\title{
Creating Interactive Digital Content: A Cross- Cultural Approach to Develop Critical Peer Feedback
}

\author{
Vidya Sarangapani \\ Open Lab \\ Newcastle University, UK \\ vidya.sarangapani@ncl.ac.uk
}

\author{
Ahmed Kharuffa \\ Open Lab \\ Newcastle University, UK \\ ahmed.kharuffa@ncl.ac.uk
}

\author{
David Leat \\ ECLS \\ Newcastle University, UK \\ david.leat@ncl.ac.uk
}

\author{
Pete Wright \\ Open Lab \\ Newcastle University, UK \\ p.c.wright@ncl.ac.uk
}

\begin{abstract}
Giving and receiving critical feedback are key 21st century learning skills, which form an integral part of enquiry based approaches to learning. Yet learners face several barriers to acquiring those skills; a lack of interest in peer-created content; social politeness; and under-valuing peerfeedback. Additionally, the perception of one's peers as equals rather than experts engenders low engagement in peer-feedback activities. In this study, we investigate and address some of these barriers through a 3-month cross-cultural activity with 60 students in two schools - in India and England. By introducing an interactive content-creation tool to facilitate the peer feedback process in a cross-cultural, Project-Based Learning (PBL) activity we initiate and utilise the cultural expertise of the students. The activity involved creating interactive content to stimulate critical feedback exchange between students where the students were considered to be 'experts' in their cultural setting. The three-month activity demonstrates that the use of an interactive contentcreation tool in a culture-sensitive PBL process aids the development of critical peer feedback and helps students to achieve a deeper understanding of their own culture and that of the 'other'.
\end{abstract}

Technology and Learning, Cross-Cultural, Interactive Content, Peer-Feedback

\section{INTRODUCTION}

Schools are responding to the 21st century skills framework by raising the importance of technologyenhanced learning and critical thinking skills in their curricula (Voogt \& Roblin, 2012). Educators acknowledge that peer feedback impacts critical thinking and are increasingly integrating technologies to support feedback exchange between peers (Kulkarni, Bernstein, \& Klemmer, 2015; Shannon, Hammer, Thurston, Diehl, \& Dow, 2016; Tinapple, Olson, \& Sadauskas, 2013).

However, factors such as students using same resources to learn, sharing similar demographics (Gerber, Olson, \& Komarek, 2012), reticence in accepting a peer's judgement due to their limited expertise (Gielen, Peeters, Dochy, Onghena, \& Struyven, 2010) and the reluctance to provide negative feedback so as to not upset the peers (Lin \& Yang, 2011) affect the quality of feedback. Furthermore, low task engagement is also shown to impact students' involvement with the feedback process (O'Dowd \& Waire, 2009).

Culture is constituted by notions of identity, race, heritage, traditions, rituals, objects, historical events, and involves interactions, values, thinking, believing, reading and writing. Lived experiences situated in a particular culture helps one to develop cultural expertise. Shared patterns of socialization impacts effectiveness of peer groups; thus, culture affects peer response (Nelson \& Carson, 2006). In our study, we entwine culture and cultural expertise through the participation of students from two schools in India and England, who also act as domain experts of their respective culture. The presence of domain experts created essential gravitas and momentum to support peer feedback exchange.

Project-Based Learning (PBL) is a constructivist approach to learning that grounds new knowledge in experiences from real life (Kaldi et al., 2011). Studies have highlighted that technology supported PBL activities enhance creativity and develop critical thinking skills (Bell, 2010; Kaldi, Filippatou, \& Govaris, 2011). If PBL can be considered as a series, feedback usually forms its final stage (Bell, 2010). Moreover, PBL activities support collaborative inquiry by encouraging students to make relevant links between information, presenting conclusions and actively seeking feedback from a real-world audience (Leat, 2017). Entwining relevant digital technologies with pedagogies such as PBL, supports the enquiry process by enabling dialogue and representation of ideas between students, teachers and the realworld communities (Kahn \& Rourke, 2005). 
In our work, we explore how an interactive contentcreation digital tool and a culturally driven PBL activity could support students to offer and receive critical peer-feedback. Additionally, it explores how context (in our case culture) could be utilised to structure and develop peer expertise.

We undertook a three-month international study and linked two schools in India and England. A culturally receptive PBL activity was designed and students were tasked to create a project around a topic that related to their peers' culture (in order to ensure that the feedback to the content is from an expert of that culture). Students were required to conduct research in the real world and generate interactive digital tasks. Subsequently, students exchanged the digital tasks with each other and sought peer feedback. Our findings highlight that interactive content-creation tools provide avenues to develop engaging tasks that influence critical peer-feedback and peer-expertise, particularly in a culturally-driven real-world setting.

Our work makes three contributions to the $\mathrm{HCl}$ community. First, we report on an empirical study that engaged students in a critical feedback exchange after a process of interactive digital content creation and consumption. Second, we explore culture as context in our study and recruit context-experts to induce rigor in the peer feedback process. Finally, we present insights on how the different elements of the study design - crosscultural context, peer experts and the creation/consumption of interactive digital content influences the level and type of feedback alongside the response and interpretation of it. We propose that the use of interactive content-creation tools along with context-informed (our case being culture-sensitive context) PBL can support critical peer feedback and help students to develop a deeper understanding of their own culture and the cultural other.

\section{RELATED WORKS}

There is growing interest within the $\mathrm{HCl}$ community to explore the impact of digital technologies within cross-cultural contexts (Heintze, Krämer, Foster, \& Lawson, 2015) and peer-feedback (Shannon et al., 2016; Wauck et al., 2017).

\section{Technology supported PBL and peer feedback}

Published work on peer feedback through PBL approaches (Ching \& Hsu, 2013; Wauck et al., 2017) have been predominantly on passive content, with no hands-on engagement with the digital content. Wauck et al. (Wauck et al., 2017) explored the impact of peer-feedback and external feedback in a project-based design course. Working with 127 undergraduate and graduate university students for nine weeks they looked at feedback from classroom peers and external crowds on their website prototype. Classroom peers provided feedback via emails whereas feedback from external crowds was amassed through social networking website/online communities or through a paid task master (MTurk). Authors mention that the study was unable to detect the perceived quality of the feedback or any actions being taken as a result of the feedback and call for research to explore on process-oriented and conceptual feedback.

Similarly, Ching and Hsu (Ching \& Hsu, 2013) worked with 21 geographically dispersed master's level university students looking at the role of peerfeedback in an online PBL environment. Participants collaborated on the online learning platform Moodle to solve instructional design problems. Feedback was provided on the designdocument and participants were required to respond to the suggestions, questions or comments received. Authors highlighted that students were mostly supportive of their peer's work and rarely criticized it. Even in areas where they had to pinpoint problems they used disclaimers or gentler tones. Authors call for more work on constructive feedback through tasks and implementation of feedback received to evidence its impact.

Shannon et al. (Shannon et al., 2016) worked with 53 graduate and undergraduate university students through a project-based innovation course where students provided feedback to their peers on group presentations and their business model document regarding a web-based evaluation system for inclass peer feedback. The study reported challenges relating to quality issues on feedback received and called for future system to address task-focused peer feedback.

Our work contributes to this thread of research by exploring how interactive content could support conceptual, task-focused, process-oriented feedback as well as the implementation of the feedback obtained from peers.

\section{Technology, cross-cultural context and peer feedback}

The role of technology to support feedback processes within cross-cultural context has also gained significant interest (Cheng, Yang, \& Andersen, 2017; Lee \& Markey, 2014; Ware \& O'Dowd, 2008). Cheng et al.(Cheng et al., 2017) worked with 68 American university students through a virtual reality game Crystallize to help them learn Japanese language. This study reported that the use of technology-led activity was unable to evidence any learning outcome and called for more work to explore how context and feedback could support learning of cultural behaviors. 
Lin and Yang (Lin \& Yang, 2011) worked with 32 second-year university students in Taiwan looking at the role of technology and peer feedback on English writing skills. Students were required to write a short piece between 120-150 words and publish their writing on a wiki-based online platform and seek peer feedback. This single institution study looked at passive online content and called for more work to understand student motivation to provide peer feedback and how peer feedback could aid in the development of reflective skills.

Ware and O'Dowd (Ware \& O'Dowd, 2008) conducted a year long, two-phase activity investigating language development in postsecondary students in Spanish and English. Through a tele-collaborative project across twophases in US, Spain and Chile, 98 students (ages 19-22) were required to write at least 300 words in their second language and share it with their peers for corrective feedback. Students used Blackboard, an on-line course management system to collaborate and provide feedback. This study also focused on peer feedback on passive content with a authors calling for future work to explore how feedback impacts technology mediated tasks alongside exploring the role of tasks to promote intercultural learning.

Lee and Markey (Lee \& Markey, 2014) developed a three-week intercultural exchange project with 28 university students from USA and Spain. Students produced a blog and a podcast and sought peer feedback on the content and language used in the blogs and podcasts. Through Blackboard, Moodle and emails students interacted with their crosscultural peers. Likewise, this study also focused on peer feedback on passive content with the authors calling for more work in looking at native informants to be a part of intercultural exchanges and to ascertain how feedback improves skills of the learner.

Bradley (Bradley, 2014) worked with 42 Swedish and American university students on a intercultural peer reviewing study to improve students' technical writing on a wiki platform. Students shared texts between each other and sought peer feedback. Authors highlight how participants found the peer feedback exchange in intercultural environment a challenging experience where comments were withheld or paraphrased to maintain relationships. Authors mention that in designing learning environment for intercultural exchange thought must be given to support feedback formulation and feedback engagement through action.

Based on the above discussion we see that there is been some work within university settings but very little has been done to understand students in school settings. Furthermore, the work focussed primarily to enhance language skills in the participants. Moreover, existing peer feedback systems employed in cross-cultural contexts have narrow types of interaction due to use of passive content. We add that the creation and consumption of interactive content in cross-cultural contexts can enhance critical peer feedback and in the process address challenges related to task engagement and intercultural learning.

\section{METHODOLOGY}

We chose to explore this research within crosscultural contexts and linked two schools across two continents. We worked with a total of 60 students (ages 11-13), 30 students in India and 30 students in England for a period of three months.

\section{Planning}

The planning phase involved setting up preliminary meetings where teachers in India and England came face-to-face on Skype to discuss the project. Following the Skype meetings, teachers in India (Sch_Ind) chose the topic 'UK and its culture' and English teachers (Sch_Eng) chose 'India and its culture'. The topics reflect our vision to harness and develop the role of the 'peer-expert', our belief being that students in a particular country would be more aware of their own cultural setting i.e. a relative expert on their culture. We felt that students could employ this cultural expertise to provide rich peer-feedback on interactive content developed by their cultural counterparts in the other country.

\section{Research}

Working in groups, students engaged in the PBL activity by first identifying a question around the topic and then conducting research to find answers to the above question. Students talked to members in their community and used the internet to conduct their research. Community here comprised of the students' parents, grandparents, relatives and friends who provided real-world information pertaining to the inquiry. Students then used the information to illustrate their learning through a digital artefact and to formulate peer-feedback. They presented their findings - in this case an interactive content-driven digital artefact described in detail below. Finally, they engaged peer feedback exchange.

\section{Digital Technology}

Following the real-world research students used the Thinking Kit (TK) ("Thinking Kit," n.d.) platform to develop a digital mystery (Kharrufa, Leat, \& Olivier, 2010a).TK is the authoring component of the digital learning application Digital Mysteries (DM).

DM is a multi-user application, the use of which is shown to promote collaborative working and higher order thinking skills (Kharrufa, Leat, \& Olivier, 
$2010 \mathrm{~b}) . \mathrm{DM}$ is the digital interpretation of the paperbased mysteries (Leat \& Nichols, 2000) approach where students manipulate a number of slips of paper containing facts, information and red herrings to answer an open-ended question. DM combines digital affordances to a mystery task, where students manipulate digital slips of clues to answer an open-ended question (Figure 1).

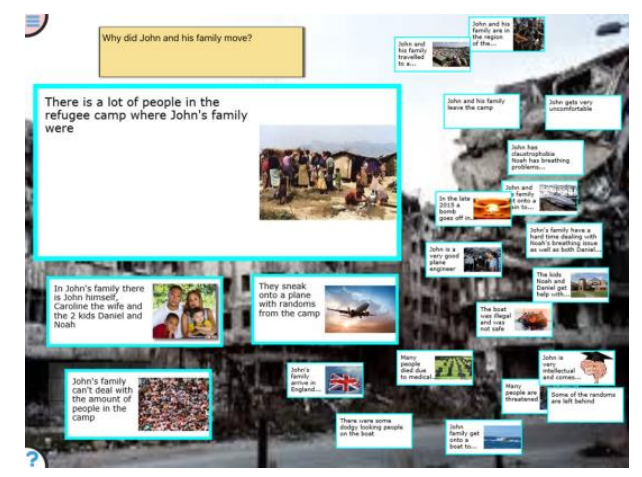

Figure 1: A digital mystery created by a student with an open-ended question 'Why did John and his family move?' and number of clues to answer the question.

Clues can be enlarged by tapping on it.

Users can create their own mystery using the TK web-interface. Using the code attached to each mystery one can download and solve it on tablets. The mysteries can be created on the TK webplatform through any internet-connected device, but solving the mystery can only be performed on a tablet through the Thinking Kit App. An exploratory study with school students $(n=12)$ focusing on the authoring process of digital mysteries highlighted that students develop cognitive skills in a non-linear manner due to the abstract nature of the authoring tool alongside demonstrating good metacognitive insights into their tasks and technology use (Heslop, Preston, Reid, \& Kharrufa, 2017).

Key features like content-creation, critical engagement and ease of activity exchange helped us to decide on this digital technology.

\section{Content-Creation}

In having to create their own mysteries, students were required to develop their own open questions relating to the task. Students undergo a process of gathering information, creating coherent interpretations of their information and finally externalized their coherency through several short bits of interactive learning content (Figure 2).

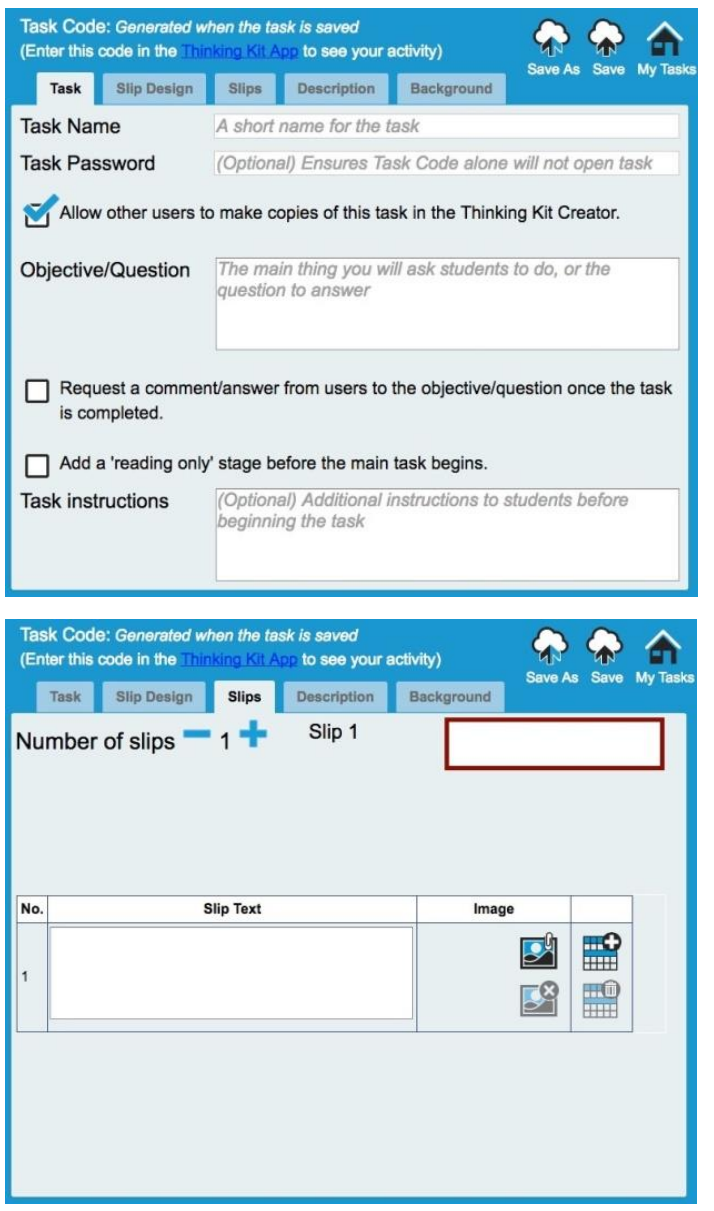

Figure 2: Dialog boxes that aid students to create clues for the digital mystery.

\section{Critical Engagement}

The collaborative use of digital mysteries also makes it possible for students to rationalize their decision-making to their peer or teacher. Several affordances in TK supports this and can be noticed when a group is trying to solve a mystery. One such action is the use of sticky tapes (Figures 3,4) which is used to link clues and highlight if the relationship is normal (rectangular sticky tape) and/or causal/temporal (arrow shaped sticky tape). Or the use of named groups (Figures 3,4) (used to classify and sort their clues) and post-it notes (used to add comments or extra information) both of which encourages discussion and decision making. Such affordances trigger task-related discussions, ensure visibility of students' thinking which inturn creates further discussion among the students as well as allowing observers to engage in the feedback process. 


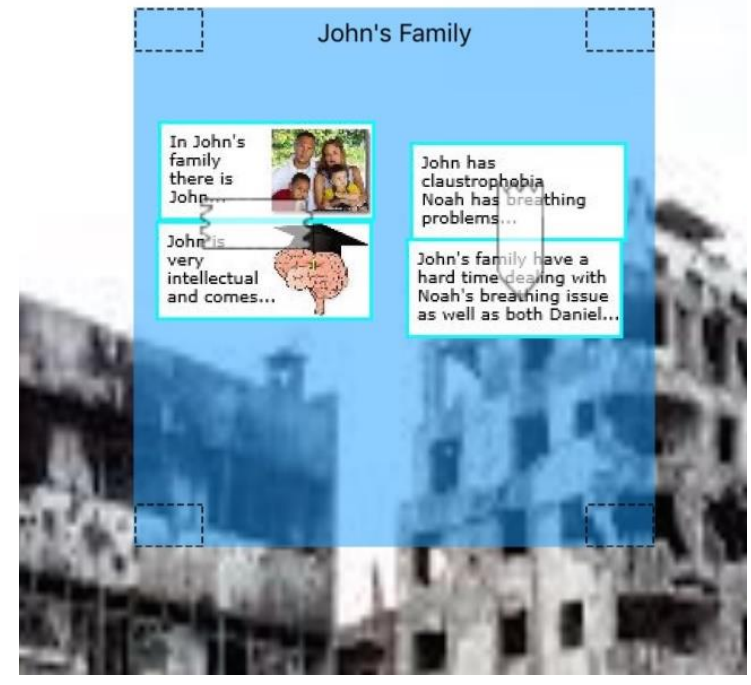

Figure 3: The 'Named Group' is John's Family. The two sticky tapes used to hold the clues together show that the relationship between the clues. Rectangular sticky tape have normal relation and arrow shaped sticky tape have causal relation.

\section{Ease of activity exchange}

Upon creating and saving the mystery a unique mystery number is generated. This number can be shared by both students and teachers with anyone in the world who have access to TK app. One can download and solve mysteries and answer the open-ended question (Figure 4). The contentcreation tool thus provides opportunity for students to show their peers how they have engaged with the feedback received.

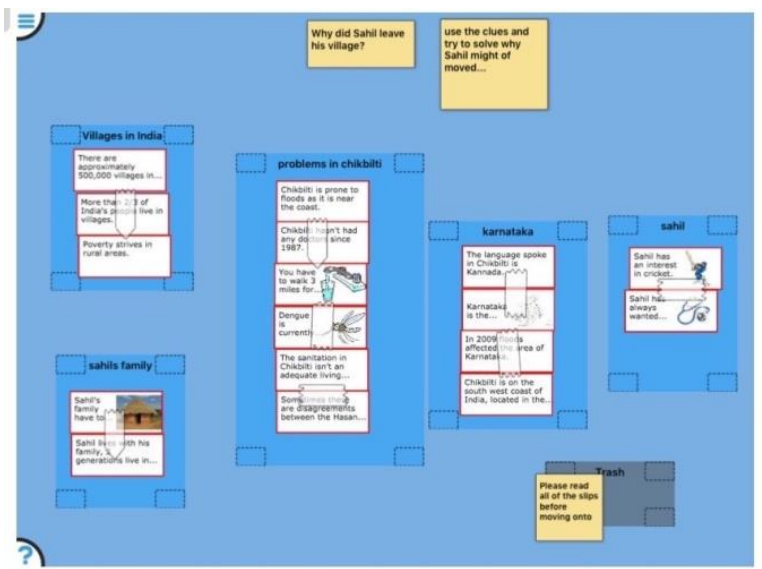

Figure 4: A digital mystery solved by a student

\section{Feedback Exchange}

This was the final phase of the project, where students exchanged the digital mysteries with each other and sought peer feedback. Students in the two schools (Sch_Ind, Sch_Eng) generated a total of 18 mysteries between themselves (Figure 5). The researcher engaged in brief discussions with the students on what constitutes 'good' feedback to support them in generating rich and relevant feedback. Students were first required to generate a reasoned answer to their peer's mystery and provide feedback in the form of a short hand-written note. Students had to provide feedback to their peers in order to view the feedback on their work. The researcher coordinated the feedback exchange including the logistics of collecting and distributing the handwritten feedback to the students in the two countries.

\begin{tabular}{|l|l|}
\hline Sch_Ind & Sch_Eng \\
\hline What happened? & George in India \\
\hline The need to immigrate & David \\
\hline Journey to England & Sanjay \\
\hline From Scotland to India..Andrew's journey & Why did Sahil leave his village? \\
\hline Arin's Future Career & Why does Sita still live with her parents? \\
\hline $7 / 7$ Bombings & Kahn \\
\hline Natasha's Dream & \\
\hline The Guitar Thief & \\
\hline Economy of UK & \\
\hline Spoons 'n' Forks & \\
\hline Landmarks of England & \\
\hline One love - One Direction & \\
\hline
\end{tabular}

Figure 5: Mysteries developed by students in India (Sch_Ind) and England (Sch_Eng)

\section{Data Collection and Analysis}

The study lasted 7 weeks in the English school (Sch_Eng) and 5 weeks in the Indian school (Sch_Ind).

A researcher was present and worked with the students through all sessions of the study. During each study visit, data was collected in the form of observational notes and photographs. Digital mysteries that were created and solved also formed a part of the data set to inform our findings.

Focus-group interviews were conducted with the students in the two schools. In addition, interviews with the class teachers and the headteachers were also conducted to gain insights into the activity.

The interview transcripts of students were analysed through an iterative process using thematic analysis (Braun \& Clarke, 2008). The transcripts were read through several times to get an impression and sense of the totality of the data. To develop a unit of analysis, segments were identified in the transcripts that showed transitional changes (e.g: change in activity, approaches to task) and coding categories were developed by employing thematic analysis. Finally, derived categories that emerged were considered as themes.

\section{FINDINGS}

The three themes that emerged through analysis is discussed as below.

(a) Creating interactive-content 
(b) Feedback reciprocity

(c) Cross-cultural context

\section{Creating interactive-content}

Students actively engaged with the TK platform to develop their digital mysteries (Figure 5). Students mentioned that creating content for an external audience who were subject experts impacted their engagement in the cross-cultural activity.

Female_1_Sch_Ind:Actually we thought about the mystery that we had to make in our own peer group....We thought about how about this mystery, what opinions will our friends in India will have about our mystery. Because we're of the same age group (as the English students) most of our ideas, like half of it, our psychology would meet, but they (English students) would have some a little bit more advanced (on the topic).....

Female_1_Sch_Ind: Research was not actually hard...but the question (that) we had to ask...the big question. We had so many topics--not just one topic. We are coming (at) each topic, with each aspect of UK. So we had to decide should we take food or we should we take -- we had to focus on one topic. So we had confusion. First we had was food, so finally we got this about chefs, the aspiring chef.... wants to go to London and wants to find a new job. Even the lifestyle over there, if she will be affected by that.

Students mentioned that creating the mystery helped them delve deeply into the content. Students discussed that digital content-creation offered the freedom to shape information as pleased and that it was better than creating a tangible artefact like scrapbook or undertaking any other form of visual learning. Students discussed the comparison between tangible artefact like a video or scrapbook to a digital mystery as below.

Male_2_Sch_Eng: I don't like the scrapbook, it's not my type of thing. I'm not a visual learner. I've been taught how to use computers, so like the digital mystery was easier for me.... It's because I'm not a visual learner, I'm more of a...

Female_1_Sch_Eng: Practical..

Male_2_Sch_Eng:...yes, practical learner.

\section{Feedback Reciprocity}

Students acknowledged that the process of creating the mystery, receiving individual feedback on their mystery and providing personal feedback on their peer's mystery was all very stimulating.

Female_1_Sch_Eng:I think it was quite interesting really, because sort of like being able to give your opinion and then getting feedback back on it- I'm not really sure, but it seemed slightly more personal rather than just doing the mystery and then going off again...

Engaging with the mystery helped students to learn about the cultural viewpoints of their peers which helped students to frame their feedback.
Female_5_Sch_Ind: They had given us clues so we were able understand in a better way that what they think about India more particularly, so it was easy to get their points and frame our feedbacks.

A common conversation was on the use of language while providing criticism. Students mention that they had to phrase their feedback so as not hurt their peers' sentiments. Feedback was provided on both content and aesthetics of the application. Some students discuss their thoughts as follows.

Female_2_Sch_Eng: That's what we're taught so we don't want to write down, "You're not very good at this, you need to do this, you need to do this." So we were really nice, we were like, "Oh I really like this part, possibly you could do this." So we didn't realise that they - because we've always been told, "If you don't want to say anything nice then you can't say anything at all."

Female_8_Sch_Ind: I actually found that a critical approach to a person's project or mystery was not that easy. We had to give only reasonable points, and we also learned how to give someone criticism without hurting his or her feelings.

Engaging with the mysteries helped students to develop insightful feedback as they could identify knowledge gaps by looking at the content developed by their peers. They added that solving the mysteries tangibly helped them to structure the feedback they were going to provide.

Female_6_Sch_Ind: The digital mystery helped us to know what the students of UK thought of India as a country. In the feedback we told them about the wrongs they had in their mystery. We also cleared some misconceptions they had about India. The mystery helped us a lot to shape our feedback.

Students also expressed disagreement on the feedback they received and did so by revisiting their mysteries. Students critically engaged with the feedback by interrogating the rationale of the comments provided and in some cases even empathised as they worked through the comments.

Female_7_Sch_Eng: I don't understand why it's not so.... l've got information in there, saying it's wrong......And they've said that the facts aren't solid. But they're facts that I've got from the internet, and other things, which are solid. But they say that they're not solid facts.

Female_9_Sch_Eng:Yes, Yes. I think that the comment about it being negative, I don't think exactly, is something that I can really improve on. Because if it's my mystery, I can't just change the whole thing. But of course, I know like, it's very obvious. Of course I know that India's not just this big, poor place. It's just because I focused my mystery on that, but a lot of people here are saying that it's only for poor. But that's not true, because I talked about how we was going to become a doctor. And he was going to a medical school in the city, and they said that, "You just talk about the poor." But I think that kind of reflects the richer side.

Students appreciated the handwritten notes they received from their peers as a feedback. This tangible aspect of receiving feedback made the 
whole process real to the students. The importance of reciprocity was highlighted by those who did not receive any feedback. This was particularly observed when the handwritten feedback was circulated in the classroom. Those who didn't receive the feedback came up to the researcher to reconfirm if their feedback was missing.

Students contrasted the experience of receiving feedback from a peer and teacher, highlighting that communication with peers from the same age group is easier due to the ability to sync into each other's viewpoints.

Female_Sch_Ind: Receiving feedback from a teacher is nice. But it is from the perspective of a person who is way older to you. They think according to their adulthood. Receiving it from a student is different. Students are children who think in a totally different way compared to the teachers. Also, you can relate to what they say because they are closer to your age.

Students exhibited multifaceted engagement with the feedback they received. Some students reflected on the feedback to know what their peers thought about the mystery. Others discussed the actionable comments and plain remarks provided in the feedback whilst some others pondered on reshaping their entire mystery to respond to the feedback.

Female_8_Sch_Ind: Our feedback included suggestions of making our mystery a little more confusing by adding more clues and red herrings. We were also suggested to add few additional pictures along with the clues to make it more attractive. Our feedback did not have many points. But our group, for the sake of progress, still reanalysed our mystery and concluded that not much change was needed in our mystery.

Female_3_Sch_Eng: And it's focusing on poverty. So I think if I would kind of answer their requests, of making it less negative, then l'd have to maybe do a whole new one. Because I don't think I could change a lot; because mine's very much he comes from a less fortunate place in India. And he wants to go and be a doctor, so he has to move to a city. But in here, it says that they don't like that I'm using a poor place, as an example. And so I think if I could, if I would like answer their requests, I'd have to rework it. I could try and start doing that now, but I don't think I could do it in this lesson, probably.

Students discussed how its only on receiving feedback they have realized the significance of critical feedback. Reading their peer-feedback stimulated students to mentally compare and contrast it to the feedback that they had offered.

Male_1_Sch_Eng: I was way too positive for mine because I hadn't had mine so I was like are they going to be nice about mine so I'll just be nice about theirs.

Female_2_Sch_Eng: I think we should have heard our feedback and seen how critical they were before we written our feedback, because then we would have felt more relaxed if they were already critical.
Teachers in their interviews mentioned that the activity has highlighted the lack of skills in students to provide and accept critical comments. They mention that students require training on how to offer and receive critical feedback. During classroom observations the researcher noticed that that Sch_Eng teachers had to step-in to clarify the feedback from their cultural peers. Teachers had to explain it to students that criticism of the mystery is not a criticism of the person who made the mystery as some of the students felt that the comments they received were 'mean'.

\section{Cross-cultural Context}

The students solved the mystery from the role of a 'peer-expert' and in doing so they could accessed their peers' understanding of the topic. The experts themselves also developed a deeper understanding of their own culture. Two students from England recount their experience as follows.

Male_Sch_Eng: It made quite a difference to me since, well I knew that they live in India which the mystery was based on. So, it basically made me think of what I would know about more and that is more common and so on. So when we did something, myths and so on so it would be realistic.

Female_Sch_Eng:I thought it was quite interesting to see that we've sort of been taught, not only in school, but sort of, as a society, that places like, for example, India, that are all very far away and mysterious or negative places, I suppose. And that's why we were putting so much negativity into our mysteries and projects and stuff. Whereas they appear to have been told that the UK or Britain or whatever is quite positive and that's why they were being so positive and it's quite interesting to see that comparison.

Students engaged in real world research to gather information for their mysteries. This research process helped them to uncover some preconceived assumptions on the topic.

Female_8_Sch_Ind:Sometimes these things also clear the misconception we have about a place. We also had some so now we're clear. We had actually researched, there were things, because we know from our friends and maybe they know from someone else, but when we actually go to personally check these things the misconceptions are actual misconceptions and are actually cleared. Like food is not so bland, so flavours are not so good as we have in India, the spiciness and everything but now when I had a topic at school. So when I searched I found many different things and I realised that no, it's not just India. Many other countries also have a lot of vast variety of food flavours and everything.

Students developed a deeper understanding of their cultural peer by reading and reflecting on the feedback. Students discussed that the cultural contexts have an influence on providing feedback.

Female_1_Sch_Eng: I think that the culture kind of does matter because I think in India maybe honesty is taught over kindness. I know that sounds really horrible. I take 
that back, sorry. Honesty is like equal to that, and I think maybe here it's like honesty, be really polite and then be honest.

Female_2_Sch_Eng:Which I don't think is actually right, I think that the way they do it is much better more beneficial because they're a bit more honest, but maybe what they can do to make theirs better is say how we can make this better. Not just say what's wrong with it. I think what we can do is say what's wrong with it not just how it can be better.

\section{DISCUSSION}

The findings from our study provide insights as to how; content-creation encouraged students to think critically about the content they generated, receiving feedback made them to think critically about both the feedback received and feedback offered, and the cultural context encouraged deep engagement with the feedback exchange process due to the presence of subject experts.

Our results highlight that interactive contentcreation helped students in our study to access internal feedback. When effective learners engage with their learning tasks, they develop internal feedback and employ cognitive routines (Hattie \& Timperley, 2007). The internal feedback amongst students took place on three occasions - first whilst they were conducting research by collaborating with their classroom peers to develop the mystery, second during content-creation where students engaged in discussions around the content they were creating, third whilst solving the mystery itself which helped students to engage in reflection (Kharrufa, Olivier, \& Leat, 2010) and supported in building critical perspective on their peers' mystery. These three stages helped the students to formulate critical comments in their feedback (even though some of these critical comments were not communicated to their peers due to politeness).

Furthermore, our work highlights the role of interactive content in encouraging students to engage in content-creation and content-use. Creating and solving a mystery required students to develop coherent links with the information at hand, cross-referencing the information and finally reconstructing the information into an accessible artefact for learning purposes. This process helped students to be critical about their own content and that of others which eventually created opportunities for rich feedback exchange.

Such an exchange within a cross-cultural setting provided a number of interesting findings. A common feature reported (Ching \& Hsu, 2013; Lin \& Yang, 2011; Shannon et al., 2016; Ware \& O'Dowd, 2008) in peer feedback is the aspect of politeness. Students from Sch_Eng discussed their difficulty in providing criticism as they did not want to offend their peers. The socio-cultural environment thus played a vital role here as the results show a difference in the balance between honesty and politeness between the two student groups; with the students from (Sch_Eng) opting for politeness over honesty while, the feedback from Sch Ind leaned more towards honesty. However, the process also made Sch_Eng students see the value of honesty and that polite feedback by itself does not benefit their peers much. This helped them rethink their beliefs on how to give and receive feedback aiming for the right balance between politeness and honesty. Students were thus able to make connections and articulate that culture has an influence on feedback activity. This is a nuanced understanding of culture that requires reflection and abstraction, a critical thinking skill. There are potential benefits to repeating this process with the same group until a good balance of politeness-honesty is reached that could satisfy all the participants.

We linked heterogeneous groups of students in our study who were engaged in providing critical feedback to each other. We tapped into their cultural expertise and presented each other as 'peer experts' which in turn helped taskengagement and critical feedback exchange. Our study has challenged some prevalent notions such as heterogeneous groups are not enthusiastic about criticizing other students in peer feedback activities and cultural homogeneity is an important factor in successful peer feedback in group working (Nelson \& Carson, 2006). Engaging peer expertise in feedback processes also tackled some challenges highlighted in intercultural activities especially that of task-engagement and feedback exchange. (Bradley, 2014; Lee \& Markey, 2014). Culturally driven activities helped students in our study to consciously and critically understand the relationship between their own culture and that of the cultural other (Lee \& Markey, 2014).

Receiving feedback on a handwritten note highlighted aspects of tangible and virtual possessions. The importance of tangibility was noted for students who did not receive handwritten feedback. The physicality of the note seemed to excite children as it was akin to receiving a gift. Those who received it were excited and were able to share it with others, compare and discuss by taking it around. Those students who did not receive the feedback had to be content looking and reading their classmates' notes. Handwritten feedback also invited challenges such as legibility, accessibility and timeliness but advantages such as being bespoke, personal and individual seemed to outweigh the challenges(Race, 2005) in this study.

Critical feedback motivated students to revisit their mysteries and respond to the queries and comments raised. It prompted students to employ their analytical and reasoning skills as we observed them 
discussing the feedback they received and how best to respond to the comments. Students emphasized that receiving feedback helped them to view their work in ways that otherwise would not have been possible. Students were observed self-regulating their actions, a skill that is considered essential for educational growth (Voogt \& Roblin, 2012).

Real-world experts play a crucial role in facilitating critical peer feedback process as noted in our study. Students were keen to complete their task as they knew of an audience expecting to engage with their work. Consequently, the task became more than just an assignment. The learning task thus morphed into a social activity (Lin \& Yang, 2011) where students created their content with real audiences in mind.

\section{FUTURE WORK}

Students seemed to categorize feedback that highlighted the gaps in their work as negative and feedback that appreciated their efforts with no critical inputs as positive. Students required teacher's/facilitator's support to interpret the feedback beyond the binary attributes. Future work could look at how technology could assist teachers to help unpick learnings from the feedback process to ensure students develop an objective outlook to critical feedback.

Students in the study explored an abstract concept like culture and turned it into a concrete learning artefact like a digital mystery with the help of a digital tool. Whilst Thinking Kit has made this possible we wonder how digital tools could support one's learning to look at concrete topics and extrapolate them into abstract concepts. Vygotsky's (Wegerif, 2013) notion of dialogic thinking plays a key construct to develop capacity for abstract reasoning. We reckon that a carefully crafted mediated-learning (Presseisen \& Kozulin, 1992) that brings the teacher and technology closer, could enable students to use experiential narratives and convert them into abstract knowledge, helping develop malleable skills and transferable learning.

\section{CONCLUSION}

This study explored linkages to understand how interactive content-creation, cross cultural context and contextual peer experts impact critical peer feedback. Our results show that students were motivated to engage in the task and the presence of an expert peer stimulated task engagement. Our analysis points that there is broader range of factors that affect critical peer feedback. We highlight context as an important component to influence critical peer feedback exchange. Our results will help $\mathrm{HCl}$ researchers working with children to learn how to coordinate cultural contexts and peer feedback using interactive content-creation tools in a way that is most beneficial to overall learning particularly in today's volatile socio-political climate.

\section{ACKNOWLEDGMENTS}

We would like to thank the two schools in India and England along with the families, children and schoolteachers who participated in this research. We also extend our thanks to IIIT, Delhi for all the help and support. This project is funded through the EPSRC Centre for Doctoral Training in Digital Civics [EP/L016176/1]. Data supporting this publication is not openly available due to ethical consideration(s). Access may be possible under appropriate agreement. Additional metadata record can be found at http://dx.doi.org/10.17634/154300-89. Please contact Newcastle Research Data Service at rdm@ncl.ac.uk for further information or access requests.

\section{REFERENCES}

Belk, R. (1988). Possessions and the Extended Self. Journal of Consumer Research, 15(2), 139-168. http://doi.org/10.1086/209154

Bell, S. (2010). Project-Based Learning for the 21st Century: Skills for the Future. The Clearing House, 83, 39-43.

Bradley, L. (2014). Peer-reviewing in an intercultural wiki environment student interaction and reflections. Computers and Composition, 34, 8095.

http://doi.org/10.1016/j.compcom.201 4.09.008

Braun, V., \& Clarke, V. (2008). Using thematic analysis in psychology. Qualitative Research in Psychology, 3(2), 77-101.

Cheng, A., Yang, L., \& Andersen, E. (2017). Teaching Language and Culture with a Virtual Reality Game. In Proceedings of the $2017 \mathrm{CHI}$ Conference on Human Factors in Computing Systems - CHI '17 (pp. 541-549). New York, New York, USA: ACM Press. http://doi.org/10.1145/3025453.30258 57 
Ching, Y.-H., \& Hsu, Y.-C. (2013). Peer Feedback to Facilitate Project-Based Learning in an Online Environment. International Review of Research in Open and Distance Learning, 14(5), 258-276.

Gerber, E. M., Olson, J. M., \& Komarek, R. L. D. (2012). Extracurricular DesignBased Learning: Preparing students for careers in innovation. International Journal of Engineering Education, 28(2), 317-324.

Gielen, S., Peeters, E., Dochy, F., Onghena, P., \& Struyven, K. (2010). Improving the effectiveness of peer feedback for learning. Learning and Instruction, 20(4), 304-315. http://doi.org/10.1016/j.learninstruc.20 09.08.007

Hattie, J., \& Timperley, H. (2007). The Power of Feedback. Review of Educational Research, .77(1), 81112. http://doi.org/10.3102/ 003465430298487

Heintze, K. E., Krämer, N., Foster, D., \& Lawson, S. (2015). Designing Student Energy Interventions : A Cross-cultural Comparison. In British HCl 2015 (pp. 247-254). http://doi.org/10.1145/2783446.27835 75

Heslop, P., Preston, A., Reid, A., \& Kharrufa, A. (2017). Students' Perceptions of Learning Processes as Co-Authors of Digital Tabletop Activities. In British $\mathrm{HCl}$.

Kahn, P., \& Rourke, K. O. (2005). Understanding Enquiry-Based Learning. Learning, 1-12.

Kaldi, S., Filippatou, D., \& Govaris, C. (2011). Project-based learning in primary schools : effects on pupils learning and attitudes. Education 313: International Journal of Primary, Elementary and Early Years Education, 39(1), 35-47. http://doi.org/10.1080/0300427090317 9538
Kharrufa, A., Leat, D., \& Olivier, P. (2010). Digital mysteries. In ACM International Conference on Interactive Tabletops and Surfaces - ITS '10 (p. 197). New York, New York, USA: ACM Press.

Kharrufa, A., Leat, D., \& Olivier, P. (2010). Digital Mysteries : Designing for Learning at the Tabletop. In ITS'10, November 7-10, 2010, Saarbru "cken, Germany (pp. 197-206).

Kharrufa, A., Olivier, P., \& Leat, D. (2010). Learning Through Reflection at the Tabletop: A Case Study with Digital Mysteries. Proc. World Conference on Educational Multimedia, Hypermedia and Telecommunications 2010, 665674.

Kulkarni, C. E., Bernstein, M. S., \& Klemmer, S. R. (2015). PeerStudio: Rapid Peer Feedback Emphasizes Revision and Improves Performance. In )ACM Conference on Learning @ Scale - L@S '15 (pp. 75-84). http://doi.org/10.1145/2724660.27246 70

Leat, D. (Ed.). (2017). Enquiry and Project Based Learning: Students, School and Society. Routledge.

Leat, D., \& Nichols, A. (2000). Observing Pupils' Mental Strategies: Signposts for Scaffolding. International Research in Geographical and Environmental Education, 9(1), 19-35.

Lee, L., \& Markey, A. (2014). A study of learners' perceptions of online intercultural exchange through Web 2.0 technologies. ReCALL, 26(3), 281-297. http://doi.org/10.1017/S09583440140 00111

Li, L., Liu, X., \& Steckelberg, A. L. (2010). Assessor or assessee: How student learning improves by giving and receiving peer feedback. British Journal of Educational Technology, 41(3), 525-536. http://doi.org/10.1111/j.14678535.2009.00968.x 
Lin, W. C., \& Yang, S. C. (2011). Exploring students' perceptions of integrating Wiki technology and peer feedback into English writing courses. English Teaching, 10(2), 88-103.

Nelson, G., \& Carson, J. (2006). Cultural issues in peer response: Revisiting "culture." In K. Hyland \& F. Hyland (Eds.), Feedback in Second Language Writing: Contexts and Issues (pp. 42-59). New York: Cambridge University Press.

O'Dowd, R., \& Waire, P. (2009). Critical issues in telecollaborative task design. Computer Assisted Language Learning, 22(2), 173-188. http://doi.org/10.1080/0958822090277 8369

Presseisen, B. Z., \& Kozulin, A. (1992). Mediated learning: The contributions of Vygotsky and Feuerstein in theory and practice. Annual Meeting of the American Educational Research Association, 5-42.

Race, P. (2005). Using feedback to help students to learn. The Higher Education Academy, 1-11.

Shannon, A., Hammer, J., Thurston, H., Diehl, N., \& Dow, S. (2016). PeerPresents: A Web-Based System for In-Class Peer Feedback During Student Presentations. Proceedings of the 2016 ACM Conference on Designing Interactive Systems, 447458. http://doi.org/10.1145/2901790.29018 16

Thinking Kit. (n.d.). Retrieved from https://www.thinking-kit.com/

Tinapple, D., Olson, L., \& Sadauskas, J. (2013). CritViz: Web-Based Software Supporting Peer Critique in Large Creative Classrooms. Bulletin of the IEEE Technical Committee on Learning Technology, 15(1), 29-35.

Voogt, J., \& Roblin, N. P. (2012). A comparative analysis of international frameworks for 21st century competences: Implications for national curriculum policies. Journal of Curriculum Studies, 44(3), 299-321. http://doi.org/10.1080/00220272.2012. 668938

Ware, P. D., \& O'Dowd, R. (2008). Peer feedback on language form in telecollaboration. Language Learning \& Technology, 12(1), 43-63.

Wauck, H., Yen, Y., Fu, W.-T., Gerber, E., Dow, S. P., \& Bailey, B. P. (2017). From in the Class or in the Wild? Peers Provide Better Design Feedback Than External Crowds. $\mathrm{CHI}$ Conference on Human Factors in Computing Systems - CHI '17, 55805591. http://doi.org/10.1145/3025453.30254 77

Wegerif, R. (2013). Dialogic : education for the Internet age. Routledge. 